\title{
Response to: An immunohistochemical approach to detect oncogenic CTNNB1 mutations in primary neoplastic tissues
}

\author{
Hendrikus J. Dubbink ${ }^{1} \cdot$ Marla Lavrijsen ${ }^{2}$ Thierry P. P. van den Bosch ${ }^{1} \cdot$ Ron Smits $\mathbb{C}^{2}$
}

Received: 24 November 2018 / Accepted: 14 December 2018 / Published online: 30 January 2019

(c) United States \& Canadian Academy of Pathology 2019

In a recent issue of Laboratory Investigation, Akyol et al. describe an immunohistochemical approach to detect oncogenic CTNNB1 mutations in primary neoplastic tissues [1]. The $\beta$-catenin signaling pathway is one of the most commonly deregulated pathways among various types of cancers [2]. One frequently observed mechanism is oncogenic $\beta$-catenin mutations encompassing the exon 3 encoded S/T phosphorylation residues (S33-S45), making the protein more resistant to proteolytic degradation and leading to enhanced nuclear signaling. Akyol et al describe a straightforward and elegant method to identify tumors carrying such mutations using paraffin sections. We have used an identical approach to exclude familial adenomatous polyposis (FAP) carriers among children with hepatoblastoma tumors, thereby supporting the genetic counseling process [3]. The method is based on staining consecutive slides of the tumor for total and exon 3-specific $\beta$-catenin monoclonal antibodies. Tumors in which wild-type $\beta$-catenin accumulates in the nucleus will show identical staining patterns with both antibodies. In contrast, tumors with (partial) deletion or mutation of the $\beta$-catenin $\mathrm{S} / \mathrm{T}$ residues will show a negative or strongly reduced staining for the exon 3 antibody. Especially tumors carrying in-frame deletions will be more readily identified. These deletions can vary in size from small intra-exonic deletions to larger ones extending up to part of exon 2 or 4 , and because of the generally bad DNA quality from formalin-fixed tissues and contamination with DNA from non-tumor cells,

Supplementary information The online version of this article (https:// doi.org/10.1038/s41374-018-0183-8) contains supplementary material, which is available to authorized users.

Ron Smits

m.j.m.smits@erasmusmc.nl

1 Departments of Pathology, Erasmus MC, University Medical Center, Rotterdam, The Netherlands

2 Departments of Gastroenterology and Hepatology, Erasmus MC, University Medical Center, Rotterdam, The Netherlands identification of these deletions can be challenging, even in the current next-generation sequencing era.

Despite the obvious advantage of rapidly identifying tumors with $\beta$-catenin mutations using this technique, we wish to address one major concern. The currently described method gives the impression that all commonly observed exon 3 mutations can be faithfully identified. However, the authors first identified tumors with aberrant staining patterns and then retrospectively identified CTNNB1 mutations in a subset of them. Using this approach several tumors carrying alterations outside the antibody epitope can easily be missed, as these would stain positive with the exon 3 antibody. The $8 \mathrm{E} 7$ clone used by the authors, was shown to recognize residues 36-44 and provide a positive staining for the commonly observed S33 and S45 $\beta$-catenin mutations [4]. The same holds true for another commercially available exon 3 antibody (clone D13A1) [3]. In some cases both antibodies may identify tumors carrying these mutations, but this may be the result of a strong hyperphosphorylation of S37/T41, which will also preclude antibody binding. Therefore, we would recommend to at least complement it with sequencing of the S/T encoding nucleotides, if one desires to obtain the full spectrum of mutations in tumor samples.

A second most likely temporary concern is that batches of antibody are available that fail to correctly recognize the exon 3 epitope. Using a recent 8E7 batch we observed that surprisingly all $\beta$-catenin amino-acid alterations are readily detected (Supplemental Figure). This pattern is highly reminiscent of the similarly named clone $8 \mathrm{E} 4$, as reported by van Noort et al. [5]. Possibly there has been a temporary mix-up of antibodies, which likely will be solved by the company, but it highlights the need for proper evaluation of antibody specificity.

When keeping these potential pitfalls in mind, we believe this novel immunohistochemical approach represents a powerful and affordable technique to rapidly identify $\beta$-catenin mutant tumors, especially the ones carrying deletions. If desired, it can also be extended by available antibodies specifically recognizing other epitopes such as S45. 


\section{Compliance with ethical standards}

Conflict of interest The authors declare that they have no conflict of interest.

Publisher's note: Springer Nature remains neutral with regard to jurisdictional claims in published maps and institutional affiliations.

\section{References}

1. Akyol A, Guner G, Ozseker HS, Isik A, Atci O, Uzun S, et al. An immunohistochemical approach to detect oncogenic CTNNB1 mutations in primary neoplastic tissues. Lab Invest 2019;99:128-37.
2. Zhan T, Rindtorff N, Boutros M. Wnt signaling in cancer. Oncogene. 2017;36:1461-73.

3. Dubbink HJ, Hollink I, Avenca Valente C, Wang W, Liu P, Doukas $\mathrm{M}$, et al. A novel tissue-based $\beta$-catenin gene and immunohistochemical analysis to exclude familial adenomatous polyposis among children with hepatoblastoma tumors. Pediatr Blood Cancer 2018;65:e26991.

4. van Noort M, Meeldijk J, van der Zee R, Destree O, Clevers H. Wnt signaling controls the phosphorylation status of beta-catenin. J Biol Chem. 2002;277:17901-5.

5. van Noort M, Weerkamp F, Clevers HC, Staal FJ. Wnt signaling and phosphorylation status of beta-catenin: importance of the correct antibody tools. Blood. 2007;110: 2778-9. 